\title{
Antifungal resistance and predominance of virulence determinants among Candida albicans isolated from various clinical specimens
}

\author{
Houdaii H. El-Houssaini ${ }^{\text {a }}$, Walid F. Elkhatib ${ }^{\text {b, }}{ }^{*}$, Omnia M. Elnabawy ${ }^{\text {d }}$, Hebatallah A. Nasser ${ }^{\mathrm{a}}$ \\ ${ }^{a}$ Department of Microbiology and Public Health, Faculty of Pharmacy, Heliopolis University for Sustainable \\ Development, 3 Cairo-Belbeis Road, El Horreya, Cairo 11788, Egypt \\ ${ }^{b}$ Microbiology and Immunology Department, School of Pharmacy \& Pharmaceutical Industries, Badr University in \\ Cairo (BUC), Entertainment Area, Badr City, Cairo, Egypt \\ ${ }^{c}$ Microbiology and Immunology Department, Faculty of Pharmacy, Ain Shams University, Abbassia, Cairol1566, \\ Egypt \\ ${ }^{d}$ Department of Clinical Pathology, Faculty of Medicine, Ain Shams University, Abbassia Square, Cairo, Egypt
}

\begin{abstract}
Candida albicans remains the most common cause of hospital-acquired fungal infections due to its virulence determinants. Resistance to antifungal therapy has increased dramatically, narrowing the few available therapeutic options due to their potential toxicity. However, the association between $C$. albicans virulence determinants and resistance profiles needs further investigation. $C$. albicans $(\mathrm{n}=25)$ isolated from various clinical samples were identified. Antibiogram analysis of the tested isolates against different antifungal agents was performed and their minimum inhibitory concentrations (MICs) were verified. Virulence determinants including extracellular hydrolytic enzymes, biofilm formation, and cell surface hydrophobicity (CSH) were investigated. Correlations between virulence determinants and resistance profiles of the experimented isolates, in addition to their potential association with the source of clinical specimens, were analyzed. All isolates were amphotericin B, nystatin and micafungin sensitive, while $100 \%$ were clotrimazole, fluconazole and voriconazole resistant. Extracellular hydrolytic activities were detected in 52, 68 and $100 \%$ of the tested isolates for phospholipase, protease, and hemolysin, respectively, while CSH and biofilm production was shown in 24 and $20 \%$ of isolates, respectively. CSH had significant $(\mathrm{P}<0.05)$ positive as well as negative associations with amphotericin $\mathrm{B}$ and fluconazole MICs, respectively. Source of clinical isolates showed significant $(\mathrm{P}<0.05)$ influence on some resistance and virulence patterns.
\end{abstract}

Keywords: Candida albicans; resistance; Virulence; correlation; source.

*Correspondence | Walid F. Elkhatib, Microbiology and Immunology Department, Faculty of Pharmacy, Ain Shams University, Abbassia, Cairo11566, Egypt. Email: walid-elkhatib@pharma.asu.edu.eg; walid.elkhatib@buc.edu.eg

Citation | El-Houssaini HH, Elkhatib WF, Elnabawy OM, Nasser HA. 2019. Antifungal resistance and predominance of virulence determinants among Candida albicans isolated from various clinical specimens. Arch Pharm Sci ASU 3(1): 11-20

DOI: $10.21608 /$ aps.2019.20210

Online ISSN: 2356-8380

Online ISSN: 2356-8380. Print ISSN: 2356-8399.

Received 05 March 2019. Accepted 23 April 2019.

Copyright: ${ }^{{ }^{2}} 2019$ El-Houssaini et al., this is an open-access article licensed under a Creative Commons Attribution 4.0 International License (CC BY 4.0), which permits unrestricted use, distribution, and reproduction in any medium, provided the original author(s) and source are credited.

Published by: Ain Shams University, Faculty of Pharmacy

\section{INTRODUCTION}

Candida albicans is part of the normal human microbiome and considered to be a harmless commensal [1]. However, within a few decades, candidiasis has become more frequent resulting in a wide array of superficial and deep infections [2] that range from those affecting the skin, mouth and vagina to systemic ones especially in 
immuno-compromised, HIV, chemotherapy and organ transplant patients [1], which have eventually led to medical and economic public health burdens [3].

In Candida species, extracellular hydrolases are essential for nutrient acquisition, adherence, colonization, host tissue penetration, invasion, dissemination and evasion from host immune responses [4]. Moreover, biofilms are associated with soaring rates of resistance to environmental stresses, as well as altered gene expression profiles [5].

Antifungal agents available for candidiasis management include polyenes, azoles, fluoropyrimidines, and the recently developed echinocandins, with the latter being used as an alternative for infections resistant to the previously mentioned antifungal agents [6]. Antifungal drug resistance has emerged as a consequence of the extensive antifungal prescription and the insufficient resources concomitant with the escalating mycotic infections, which occurs through different mechanisms such as upregulation of drug efflux pumps leading to a reduced intracellular antifungal concentration, qualitative or quantitative changes in the drug targets as well as metabolic alterations [7].

This study aimed to appraise the antifungal resistance as well as virulence determinants comprising secreted hydrolases, $\mathrm{CSH}$ and biofilm production, as well as to evaluate the association between these phenotypes among $C$. albicans isolated from several clinical samples in Egyptian patients.

\section{MATERIAL AND METHODS}

\subsection{C. albicans isolates}

In the current study, $C$. albicans $(\mathrm{n}=25)$ were isolated from various clinical samples; urine $(n=$
11), sputum $(n=9)$, drain $(n=2)$, pus $(n=1)$, wound $(n=1)$ and blood $(n=1)$, which were collected from El-Demerdash hospital, Ain Shams University (Cairo, Egypt) during 2015. Identification and maintenance of isolates were carried out as previously described [8], [9, 10].

\subsection{Antifungal resistance profile}

Antibiogram analysis against nystatin (NYS), amphotericin B (AMB), fluconazole (FLU), voriconazole (VOR), clotrimazole (CLT) and micafungin (MCF) for the tested isolates was carried out by the broth microdilution method and the MICs were interpreted according to the Clinical and Laboratory Standards Institute (CLSI) M27-A3 protocol recommendations [10, 11 .

\subsection{Phospholipase assay}

Production of phospholipase by $C$. albicans was assessed by the plate method described by $[10,12,13]$. The phospholipase index $\left(\mathrm{P}_{\mathrm{z}}\right)$ was expressed as the ratio of the colony diameter to the precipitation zone diameter. $\mathrm{A}_{\mathrm{z}}$ value of less than 1 refers to phospholipase activity by the tested isolate [13].

\subsection{Aspartyl protease assay}

Production of secreted aspartyl protease by $C$. albicans was evaluated by the plate method described by $[10,14]$. The protease index $\left(\operatorname{Pr}_{z}\right)$ was expressed as the ratio of the colony diameter to that of the unstained zone of proteolysis. $\mathrm{A} \mathrm{Pr}_{\mathrm{z}}$ value of less than 1 refers to aspartyl protease activity by the tested isolate [15].

\subsection{Haemolysin assay}

Haemolysin production by $C$. albicans was determined by the plate method previously described by $[\mathbf{1 0}, 16]$. Hemolytic activity $\left(\mathrm{H}_{\mathrm{z}}\right)$ was expressed as the ratio of colony diameter to

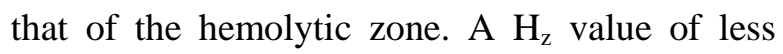
than 1 refers to hemolytic activity by the tested isolate [15]. 


\subsection{Cell surface hydrophobicity}

The microbial adhesion assay to hydrocarbons (MATH) modified after [10, 17] was used to evaluate the cell surface hydrophobicity (CSH) of the tested isolates using xylene. The hydrophobicity percentage was calculated using the following formula: cell surface hydrophobicity $(\%)=\left[1-\left(\mathrm{A}_{1} / \mathrm{A}_{0}\right)\right] \times 100$. The hydrophobicity levels were classified as hydrophilic, moderately hydrophobic and strongly hydrophobic [18].

\subsection{Biofilm formation}

Production of biofilm was assessed using the crystal violet method previously described by $[10,19,20]$. The medium used for the biofilm assay was Sabouraud's dextrose broth (SDB) (Oxoid LTD., U.K) supplemented with glucose $(8 \% \mathrm{w} / \mathrm{v})$ to promote biofilm formation. The OD cut-off value (ODc) for biofilm production was described as three standard deviations above the mean absorbance of the yeast-free negative control and accordingly, the isolates were classified as none, weak, moderate and strong biofilm producers [21].

\subsection{Statistical analysis}

The current study's statistical analyses were completed with the SPSS version 22.0 statistical software. Non-parametric tests were carried out and the quantitative variables were designated as a minimum, median and maximum. Using the Spearman's rank correlation, the association between median MICs and virulence determinants was conducted and expressed in terms of Spearman's correlation coefficient $\left(r_{s}\right)$. Differences between median values of quantitative variables were checked among the different sources groups using the Kruskal Wallis test [10]. The $p$-values of less than 0.05 were reckoned statistically significant in all tests.

\section{RESULTS}

\subsection{Identification of Candida albicans}

Typical $C$. albicans germ tubes were seen under a light microscope after human serum inoculation and incubation for $2-4 \mathrm{~h}$ at $37{ }^{\circ} \mathrm{C}$ for all the tested isolates $(n=25)$. C. albicans isolates identification was confirmed using the Candida Chromogenic agar, which appeared as green colonies for all the tested isolates $(n=25)$.

\subsection{Antifungal resistance profiles}

Results of the antibiogram analysis revealed that $C$. albicans isolates demonstrated high resistance rates to CLT $(92 \%)$ (MIC $\geq 1 \mu \mathrm{g} / \mathrm{mL}$ ), VOR $(100 \%)(\mathrm{MIC} \geq 1 \mu \mathrm{g} / \mathrm{mL})$ and FLU $(100 \%)$ (MIC $\geq 8 \mu \mathrm{g} / \mathrm{mL}$ ). On the contrary, $100 \%$ of the isolates showed susceptibility to MCF (MIC < $0.5 \mu \mathrm{g} / \mathrm{mL}), \mathrm{AMB}(\mathrm{MIC} \leq 1 \mu \mathrm{g} / \mathrm{mL})$ and NYS (MIC $<8 \mu \mathrm{g} / \mathrm{mL}$ ).

When evaluating the potential association between the source of clinical specimens and the resistance profiles of $C$. albicans, results revealed that isolates recovered from sputum samples showed statistically significant $(p<0.05)$ higher levels of resistance to NYS, CLT, and MCF as well as those in the miscellaneous group to NYS in comparison to those in the urine group samples (Table 1). Furthermore, no statistically significant difference $(p>0.05)$ was observed in the resistance patterns of AMB, FLU, and VOR based on the source of clinical specimens.

\subsection{Virulence determinants assays}

C. albicans virulence determinants (secreted hydrolytic enzymes, $\mathrm{CSH}$, and biofilm formation) were evaluated in relation to the source of the clinical specimens and results were demonstrated in Fig. 1. In regards to the secreted hydrolases production, $52 \%$ of the isolates produced phospholipase with $\mathrm{P}_{\mathrm{Z}}$ varying from 0.7253 to 0.9524 , whereas secreted aspartyl protease was proven in $68 \%$ with $\operatorname{Pr}_{\mathrm{z}}$ values varying from $0.3889-0.8889$. Moreover, $100 \%$ of the isolates had hemolytic activity with $\mathrm{H}_{\mathrm{z}}$ varying from 0.4667 to 0.6866 . Concerning $\mathrm{CSH}$, the tested isolates showed variable hydrophobicity levels and were accordingly distributed into different categories, where most of them were hydrophilic (76\%) and the rest showed strong (16\%) and moderate $(8 \%)$ hydrophobic characters. Moreover, $C$. albicans isolates were grouped according to their ability to produce biofilms where $80 \%$ of isolates were non-biofilm producers and the rest demonstrated moderate $(8 \%)$ and weak (12\%) biofilm formation 
capacities. It is worthy to note that the majority of the tested isolates showing strong cell surface hydrophobicity and biofilm forming capacity were recovered from sputum samples.

Table 1. Comparison of median antifungal MICs across the different Candida albicans clinical specimens' sources

\begin{tabular}{lllllll}
\hline \multirow{2}{*}{ Source } & \multicolumn{2}{l}{ Median MICs $(\boldsymbol{\mu g} / \mathbf{m L})$} & & & & \\
Amphotericin B & Nystatin & Clotrimazole & Fluconazole & Voriconazole & Micafungin \\
\hline Urine & 0.03 & 0.25 & 32 & 128 & 32 & 0.08 \\
& $(0.03-0.06)$ & $(0.006-0.50)$ & $(2-64)$ & $(64-128)$ & $(32-32)$ & $(0.03-0.125)$ \\
\multirow{2}{*}{ Sputum } & 0.06 & 0.5 & 64 & 128 & 32 & 0.16 \\
& $(0.03-0.5)$ & $(0.25-2.00)$ & $(32-64)$ & $(64-256)$ & $(32-64)$ & $(0.06-0.125)$ \\
Miscellaneous & 0.03 & 0.5 & 32 & 128 & 32 & 0.08 \\
& $(0.03-0.125)$ & $(0.25-4.0)$ & $(32-34)$ & $(64-128)$ & $(32-32)$ & $(0.06-0.125)$ \\
& & & & & & \\
P value & 0.212 & 0.004 & 0.004 & 0.619 & 0.411 & 0.026 \\
\hline
\end{tabular}

*p-values of less than 0.05 were considered statistically significant. Minimum and maximum values are presented between brackets. MICs: Minimum inhibitory concentrations

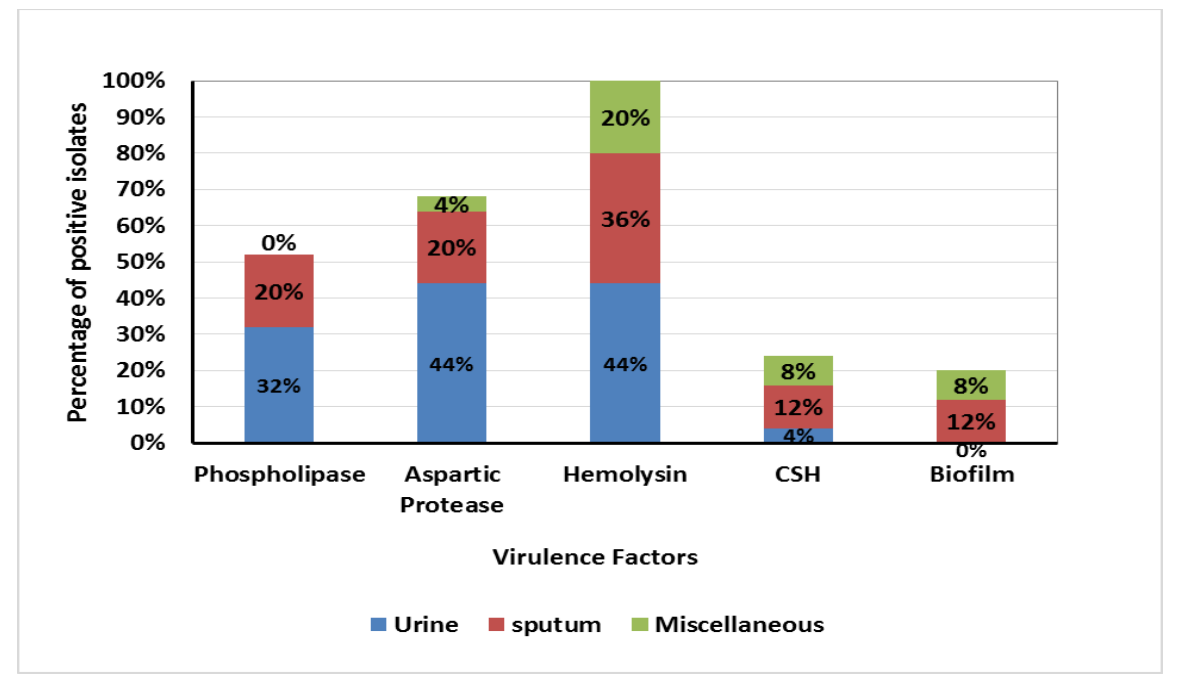

Fig. 1. Percentage of virulence determinants production (phospholipase, an aspartyl protease, hemolysin, cell surface hydrophobicity, and biofilm formation) by Candida albicans isolates with respect to the source of clinical specimens

When evaluating the potential relationship between the source of clinical specimens and virulence determinants of the isolates, the results revealed that $C$. albicans isolates recovered from urine samples had a statistically significant $(p<$
0.05) higher level of aspartyl protease production (i.e. lower Prz value), as compared to that recovered from the sputum and miscellaneous group samples (Table 2). However, no statistically significant $(p>0.05)$ difference was 
detected in the virulence patterns of phospholipase, hemolysin, $\mathrm{CSH}$, and biofilm relatively to the source of clinical specimens.

\subsection{Correlation between antifungal resistance profiles and virulence determinants}

Correlation between $C$. albicans antifungal resistance profiles and virulence attributes is shown in Table 3. Correlation coefficients highlighted significant $(p<0.05)$ positive $\left(\mathrm{r}_{\mathrm{s}}=\right.$ $0.414)$, as well as negative $\left(r_{s}=-0.439\right)$ associations between CSH and AMB and FLU MICs, respectively.

Table 2. Comparison of median values of virulence factors across Candida albicans clinical specimens' sources

\section{Source of clinical specimen}

\begin{tabular}{|c|c|c|c|c|}
\hline Virulence Factor & $\begin{array}{l}\text { Urine } \\
(\mathrm{n}=11)\end{array}$ & $\begin{array}{l}\text { Sputum } \\
(\mathrm{n}=9)\end{array}$ & $\begin{array}{l}\text { Miscellaneous } \\
\qquad(\mathrm{n}=5)\end{array}$ & $p$ value \\
\hline Phospholipase (Pz) & $\begin{array}{c}0.8889 \\
(0.7326-1.0000)\end{array}$ & $\begin{array}{c}0.8586 \\
(0.7253-1.0000)\end{array}$ & $\begin{array}{c}1.0000 \\
(1.0000-1.0000)\end{array}$ & 0.057 \\
\hline Asp. Protease (Prz) & $\begin{array}{c}0.6143 \\
(0.3889-0.8889)\end{array}$ & $\begin{array}{c}0.8194 \\
(0.4292-1.000)\end{array}$ & $\begin{array}{c}1.0000 \\
(0.4792-1.000)\end{array}$ & 0.043 \\
\hline Haemolysin $(\mathrm{Hz})$ & $\begin{array}{c}0.5503 \\
(0.4848-0.6458)\end{array}$ & $\begin{array}{c}0.5556 \\
(0.4667-0.6866)\end{array}$ & $\begin{array}{c}0.5707 \\
(0.5185-0.6345)\end{array}$ & 0.624 \\
\hline Cell surface hydrophobicity & $\begin{array}{c}9.6296 \\
(2.8700-20.4684)\end{array}$ & $\begin{array}{c}8.5685 \\
(5.5000-90.7870)\end{array}$ & $\begin{array}{c}15.6371 \\
(5.0830-86.3283)\end{array}$ & 0.498 \\
\hline Biofilm $\left(\mathrm{OD}_{590}\right)$ & $\begin{array}{c}0.03733 \\
(0.0155-0.0683)\end{array}$ & $\begin{array}{c}0.0525 \\
(0.007-0.450)\end{array}$ & $\begin{array}{c}0.034 \\
(0.009-0.378)\end{array}$ & 0.832 \\
\hline
\end{tabular}

\footnotetext{
* $p$-values of less than 0.05 were considered statistically significant. Minimum and maximum values are presented between brackets. Pz, Phospholipase index; Prz, Protease index; Hz, Haemolytic activity; OD, Optical density.
} 
Table 3. Correlation coefficients $\left(\mathrm{r}_{\mathrm{s}}\right)$ between virulence factors and antifungal MICs of Candida albicans clinical isolates

\begin{tabular}{lccccc}
\hline \multicolumn{1}{c}{\begin{tabular}{c} 
Antifungal \\
\multicolumn{1}{c}{ MICs }
\end{tabular}} & Phospholipase & Asp. protease & Haemolysin & CSH & Biofilm \\
\hline Amphotericin B & 0.295 & 0.329 & -0.054 & 0.414 & 0.377 \\
& $(0.152)$ & $(0.108)$ & $(0.796)$ & $\mathbf{( 0 . 0 4 )}$ & $(0.063)$ \\
Nystatin & 0.293 & 0.210 & 0.116 & 0.095 & 0.037 \\
& $(0.155)$ & $(0.314)$ & $(0.581)$ & $(0.652)$ & $(0.859)$ \\
Clotrimazole & 0.139 & 0.241 & -0.025 & 0.340 & 0.033 \\
& $(0.508)$ & $(0.245)$ & $(0.907)$ & $(0.096)$ & $(0.875)$ \\
Fluconazole & 0.026 & -0.315 & 0.229 & -0.439 & -0.346 \\
& $(0.900)$ & $(0.125)$ & $(0.271)$ & $(0.028)$ & $(0.091)$ \\
Voriconazole & -0.210 & -0.144 & 0.170 & -0.198 & -0.326 \\
& $(0.314)$ & $(0.493)$ & $(0.417)$ & $(0.342)$ & $(0.112)$ \\
Micafungin & 0.014 & 0.208 & 0.199 & 0.129 & -0.166 \\
& $(0.948)$ & $(0.319)$ & $(0.341)$ & $(0.538)$ & $(0.429)$ \\
\hline
\end{tabular}

*p-values of less than 0.05 (in bold) were considered statistically significant. MICs: Minimum inhibitory concentrations

\section{DISCUSSION}

As the most prominent opportunistic fungal pathogen, candidiasis has increased dramatically worldwide, with the species $C$. albicans responsible for most of these infections [22]. The current study was conceived to explore the relationship between the resistance and virulence profiles of $C$. albicans isolated from various clinical samples in Egyptian patients. Antibiogram analysis against six antifungal drugs was performed and the isolates were further evaluated for production of secreted hydrolases, $\mathrm{CSH}$, and biofilm formation.

The antibiogram analysis revealed high susceptibility rates to $\mathrm{MCF}, \mathrm{AMB}$, and NYS. These observations are in accordance with some previous studies have reported susceptibility to AMB [23], NYS [24] as well as MCF [25]. However, high resistance rates were detected against CLT, FLU, and VOR. These findings are in accordance with a previous study in Portugal [23] regarding the FLU resistance, while others reported lower resistance rates for CLT and VOR such as 23\% in India [26] and 3.2\% in China, respectively. These variations in antifungal resistance profiles can be pertained to the different sources and localities of collections of the clinical specimens in addition to the rational antifungal prescription. Furthermore, some of these studies had their isolates collected from cancer and HIV patients and involved other nonalbicans Candida. It is noteworthy that azole resistance can be attributed to the overexpression or mutations in ERG11 gene, which encodes the lanosterol $14 \alpha$-demethylase enzyme [27] or overexpression of drug efflux pumps, which results in a decreased intracellular drug concentrations [28]. The current observations may also highlight the possible impact of the inappropriate antifungal use on the emergence of such massive azole resistance rates in Egypt, the 
fact that could be attributed to providing treatment for patients without performing antifungal susceptibility testing, which is needed to customize an optimum therapeutic plan for each case individually. Moreover, species identification is an essential step to provide appropriate treatment to critically ill patients [29].

The current results reflected significant associations $(p<0.05)$ between the source of clinical specimens and the resistance patterns of the tested isolates. Deorukhkar et al [30] reported that fluconazole resistance was more seen in Candida tropicalis isolates recovered from cases of candidemia, oropharyngeal candidiasis, and vulvovaginal candidiasis, while AMB resistance was more common in $C$. tropicalis recovered from blood cultures and oropharyngeal swabs. These observations suggest that the source of clinical specimens can have an impact on the susceptibility of Candida species to antifungal therapy. However, a higher number of samples is required to confirm such a conclusion.

In the current study, production of hydrolytic enzymes was evaluated as a major part of $C$. albicans pathogenicity determinants. Haemolysin, an aspartyl protease, and phospholipase were detected in 100, 68 and 52\% of the $C$. albicans isolates, respectively. Various production rates of extracellular hydrolases have been reported by other workers as $47.6 \%$, [31] 82.1\% [32] and 100\% [33] for phospholipase, aspartyl protease, and hemolysin, respectively, which emphasizes the importance of secreted hydrolases as potent $C$. albicans virulence attributes. In addition, the majority of the isolates demonstrated hydrophilic nature, while fewer isolates showed moderate and high hydrophobicity levels. These findings are in agreement with a previous report in Portugal [23], where $C$. albicans was found to have the lowest hydrophobic character among the different Candida spp. tested. Furthermore, biofilm production was evident in $20 \%$ of the tested isolates, which reflects similarities with a Swedish study [34], where C. albicans isolates showed low biofilm production in comparison to the other Candida spp., indicating that biofilm formation may be more essential for non-albicans Candida infections than the $C$. albicans ones.

The present study revealed significant associations $(p<0.05)$ between the source of clinical specimens and virulence determinants of C. albicans. In a reported study by Taylor et al [35], site-specific virulence of $C$. albicans was evident for mucosal sites, although few isolates were virulent systemically. Candida spp. have the ability to colonize and virtually infect each organ in the human body, the fact that makes it fundamental for the opportunistic pathogen to acquire a number of similar but independently regulated, functionally distinct hydrolytic enzyme genes expressed under a variety of environmental conditions in order to survive and induce infection at different body sites [36].

In an attempt to explore the potential association between $C$. albicans resistance and virulence profiles, the present study revealed significant positive and negative associations $(p<$ 0.05) between CSH and AMB as well as FLU MICs, respectively. In a previous Spanish study [37], C. tropicalis isolates, which were AMBresistant showed enlarged cell walls in comparison to AMB-sensitive ones. These findings could be attributed to higher $\beta-1,3-$ glucan levels in the cell walls of the resistant Candida isolates rendering them more hydrophobic in nature. In another report conducted by Graybill et al [38], they pointed out that compromised pathogenicity of some $C$. albicans isolates was correlated to rising fluconazole resistance, which indicates that these limited-virulence isolates may not prominently induce infection. 


\section{CONCLUSION}

The current study reported the susceptibilities of $C$. albicans isolates to various antifungal agents representing different classes and evaluation of different pathogenicity mechanisms in addition to their potential relationship between them and the source of clinical specimens. According to the current study's findings, the development of novel therapeutic tactics combining drug resistance and virulence mechanisms in candidiasis management would be recommended. Further mechanistic studies are required to explore the multi-factorial profile of gene expression in $C$. albicans clinical isolates. The current study would also encourage the use of other antifungal agents than azoles in the management of candidiasis as well as applying combination therapy in dose regimens to decelerate the escalating azole resistance rates.

\section{Acknowledgment}

The authors would like to thank Nouran Nagy, 57357 Hospital (El-Sayeda Zainab, Cairo, Egypt) for computing the present study's statistical analyses, as well as the medical staff and technicians at the main Microbiology laboratory in El-Demerdash hospital, Faculty of Medicine, Ain Shams University (Abbassia, Cairo, Egypt) for their assistance in samples collection and clinical isolates purification.

\section{Conflict of interest}

The authors declare no conflict of interests.

\section{List of abbreviations}

CLSI, Clinical and Laboratory Standards Institute; SDA, Sabouraud's dextrose agar; SAPs, secreted aspartyl proteases; NAC, non- albicans Candida; BMD, broth microdilution; MOPS, Nmorpholinepropanesulfonic acid; $\mathrm{P}_{\mathrm{z}}$, Phospholipase index; BSA, bovine serum albumin; Prz, Protease index; Hz, Haemolytic activity; MATH, microbial adhesion assay to hydrocarbons; S, susceptible; S-DD, susceptible dose dependent; I, intermediate; R, resistant; MICs, minimum inhibitory concentrations; AMB, amphotericin B; NYS, nystatin; CLT, clotrimazole; FLU, fluconazole; VOR, voriconazole; $\mathrm{MCF}$, micafungin; $\mathrm{CSH}$, cell surface hydrophobicity; OD, optical density; ODc, OD cut-off; CFU, colony forming unit, PBS, phosphate buffered saline.

\section{REFERENCES}

1. Mayer FL, Wilson D, Hube B. Candida albicans pathogenicity mechanisms. Virulence. 2013; 4: 119-128.

2. Kauffman CA, Pappas PG, Sobel JD, Dismukes WE. Candidiasis. In: Vazquez JA, Sobel JD, editors. Essentials of Clinical Mycology. Springer-Verlag: New York; 2011, p. 167-206.

3. Lai CC, Wang CY, Liu WL, Huang YT, Hsueh PR. Time to positivity of blood cultures of different Candida species causing fungaemia. J Med Microbiol. 2012; 61(5): 701-704.

4. Ells R, Kilian W, Hugo A, Albertyn J, Kock JL, Pohl CH. Virulence of South African Candida albicans strains isolated from different clinical samples. Med Mycol. 2014; 52(3): 246-253.

5. Ramage G, Rajendran R, Sherry L, Williams C. Fungal biofilm resistance. Int J Microbiol. 2012; 2012: 1-14.

6. Basma R, Barada G, Ojaimi N, Khalaf RA. Susceptibility of Candida albicans to common and novel antifungal drugs, and relationship between the mating type locus and resistance, in Lebanese hospital isolates. Mycoses. 2009; 52(2): 141-148.

7. Sanglard D. Emerging threats in antifungal-resistant fungal pathogens. Front Med. 2016; 3: 1-10.

8. Williams D, Lewis M. Oral Microbiology: Isolation and identification of Candida from the oral cavity. Oral Dis. 2000; 6(1): 3-11.

9. Al-Wasify RS. Sensitivity and specificity of chromogenic media for detection of some pathogens in water. Internet $\mathbf{J}$ Env Sustainable. 2013; 2(1): 1-9.

10. El-Houssaini HH, Elnabawy OM, Nasser HA, Elkhatib WF. Correlation between antifungal resistance and virulence factors in Candida 
albicans recovered from vaginal specimens. Microb Pathog. 2018; 128:13-19.

11. Clinical and Laboratory Standards Institute. Reference method for broth dilution antifungal susceptibility testing of yeasts; approved standard; CLSI document M27-A3. 2008; 28: 612 .

12. Samaranayake L, Raeside JM, MacFarlane T. Factors affecting the phospholipase activity of Candida species in vitro. J Med Vet Mycol. 1984; 22(3): 201-207.

13. Price MF, Wilkinson ID, Gentry LO. Plate method for detection of phospholipase activity in Candida albicans. Sabouraudia. 1982; 20(1): 714.

14. Staib F. Serum-proteins as nitrogen source for yeastlike fungi. Sabouraudia. 1966; 4(3): 187193.

15. Deorukhkar S, Saini S. Virulence Markers and Antifungal Susceptibility Profile of Candida glabrata: An Emerging Pathogen. Br Microbiol Res J. 2014; 4: 39.

16. Manns JM, Mosser DM, Buckley HR. Production of a hemolytic factor by Candida albicans. Infect Immun. 1994; 62(11): 5154-5156.

17. Rosenberg M. Bacterial adherence to hydrocarbons: a useful technique for studying cell surface hydrophobicity. FEMS Microbiol Lett. 1984; 22(3): 289-295.

18. Kadam T, Rupa L, Balhal DK, Totewad ND, Gyananath G. Determination of the degree of hydrophobicity - a technique to assess bacterial colonization on the leaf surface and root region of lotus plant. Asian J Exp Sci. 2009; 23(1): 13539.

19. Shin JH, Kee SJ, Shin MG, Kim SH, Shin DH, Lee $\mathrm{SK}$, et al. Biofilm production by isolates of Candida species recovered from nonneutropenic patients: comparison of bloodstream isolates with isolates from other sources. J Clin Microbiol. 2002; 40(4): 1244-1248.

20. Jin Y, Yip HK, Samaranayake YH, Yau JY, Samaranayake LP. Biofilm-forming ability of Candida albicans is unlikely to contribute to high levels of oral yeast carriage in cases of human immunodeficiency virus infection. J Clin Microbiol. 2003; 41(7): 2961-2967.
21. Brilhante RSN, Bittencourt PV, de Souza Collares Castelo-Branco D, de Melo Guedes GM, de Oliveira JS, Alencar LP, et al. Biofilms of Candida spp. from the ocular conjunctiva of horses with reduced azole susceptibility: a complicating factor for the treatment of keratomycosis? Vet Ophthalmol. 2017; 20: 539546.

22. Kaur R, Goyal R, Dhakad MS, Bhalla P, Kumar R. Epidemiology and virulence determinants including biofilm profile of Candida infections in an ICU in a tertiary hospital in India. J Mycol. 2014; 2014: 303491.

23. Silva-Dias A, Miranda IM, Branco J, MonteiroSoares M, Pina-Vaz C, Rodrigues AG. Adhesion, biofilm formation, cell surface hydrophobicity, and antifungal planktonic susceptibility: relationship among Candida spp. Front Microbiol. 2015; 6: 205.

24. Santa LA, Díaz LS, Illnait MZ, Perurena ML, de Francisco Cantelar N, Fernández CA. Susceptibility to nystatin of oral Candida isolates and its correlation with the response to treatment. Rev Cubana Med Trop. 2010; 62(3): 237-244.

25. Pfaller MA, Messer SA, Diekema DJ, Jones RN, Castanheira M. Use of micafungin as a surrogate marker to predict susceptibility and resistance to caspofungin among 3,764 clinical isolates of Candida by use of CLSI methods and interpretive criteria. J Clin Microbiol. 2014; 52(1): 108-114.

26. Rathod VS, Raut JS, Karuppayil SM. In vitro antifungal susceptibility reveals occurrence of azole resistance among clinical isolates of Candida albicans. Asian J Pharm Clin Res. 2012; 5(3): 170-3.

27. Henry KW, Nickels JT, Edlind TD. Upregulation of ERG Genes in Candida Species by Azoles and Other Sterol Biosynthesis Inhibitors. Antimicrob Agents Chemother. 2000; 44(10): 2693-2700.

28. Paul S, Moye-Rowley WS. Multidrug resistance in fungi: regulation of transporter-encoding gene expression. Front Physiol. 2014; 5: 143.

29. Eraso E, Moragues MD, Villar-Vidal M, Sahand IH, González-Gómez N, Pontón J. Evaluation of the new chromogenic medium Candida ID 2 for isolation and identification of Candida albicans and other medically important Candida species. J Clin Microbiol. 2006; 44(9): 3340-3345. 
30. Deorukhkar SC, Saini S, Mathew S. Virulence factors contributing to pathogenicity of Candida tropicalis and its antifungal susceptibility profile. Int J Microbiol. 2014; 2014:1-6.

31. Udayalaxmi J, Shenoy N. Comparison between biofilm production, phospholipase and haemolytic activity of different species of Candida isolated from dental caries lesions in children. J Clin Diagn Res: JCDR. 2016; 10(4): DC21.

32. Sachin C, Ruchi K, Santosh S. In vitro evaluation of proteinase, phospholipase and haemolysin activities of Candida species isolated from clinical specimens. Int J Med Biomed Res. 2012; 1(2): 153-157.

33. Sacristan B, Blanco MT, Galan-Ladero MA, Blanco J, Perez-Giraldo C, Gomez-Garcia AC. Aspartyl proteinase, phospholipase, hemolytic activities and biofilm production of Candida albicans isolated from bronchial aspirates of ICU patients. Med Mycol. 2011; 49(1): 94-97.

34. Pannanusorn S, Fernandez V, Römling U. Prevalence of biofilm formation in clinical isolates of Candida species causing bloodstream infection. Mycoses. 2013: 56(3): 264-272.

35. Taylor BN, Fichtenbaum C, Saavedra M, Slavinsky III J, Swoboda R, Wozniak K. In vivo virulence of Candida albicans isolates causing mucosal infections in people infected with the human immunodeficiency virus. J Infect Dis. 2000; 182(3): 955-959.

36. Hube B, Naglik J. Candida albicans proteinases: resolving the mystery of a gene family. Microbiology. 2001; 147(8): 1997-2005.

37. Mesa-Arango AC, Rueda C, Román E, Quintin J, Terrón MC, Luque D. Cell wall changes in AmB-resistant strains from Candida tropicalis and relationship with the immune responses elicited by the host. Antimicrob Agents Chemother. 2016; AAC. 02681-15.

38. Graybill JR, Montalbo E, Kirkpatrick WR, Luther MF, Revankar SG, Patterson TF. Fluconazole versus Candida albicans: a complex relationship. Antimicrob Agents Chemother. 1998; 42(11): 2938-2942. 